\title{
Séries de problèmes dans une tradition d'enseignement des mathématiques en Hongrie au $20^{\mathrm{e}}$ siècle $^{1}$
}

\author{
Katalin Gosztonyi ${ }^{\mathrm{a}}$ \\ Université de Szeged (Hongrie) et LDAR, Université Paris Diderot (France) \\ Université de Szeged, 1 Aradi vértanúk tere, 6720 Szeged, Hongrie
}

\begin{abstract}
Résumé. Dans cet article, nous discutons l'intérêt d'étudier des « séries de problèmes » pour la caractérisation d'une tradition d'enseignement des mathématiques en Hongrie au $20^{e}$ siècle. Dans la partie 1, nous résumons le contexte historique et les principes partagés dans cette tradition sur les mathématiques et leur enseignement. Dans la partie 2, nous étudions plus en détail un texte d'un membre de cette communauté, un extrait du Jeux avec l'infini de Rózsa Péter, qui nous servira de modèle pour les analyses ultérieures. Dans la partie 3, nous présentons différents documents liés à la réforme de l'enseignement des mathématiques dirigée par Tamás Varga dans les décennies 1960 et 1970, cette réforme étant considérée comme une étape significative dans l'histoire de cette « tradition hongroise ». Nous tenterons de montrer qu'une réflexion sur la mise en ordre des problèmes joue un rôle crucial dans la structuration de ces textes divers, écrits souvent dans une forme narrative et quasi-littéraire ; et que l'étude des « séries de problèmes » présentées dans ces textes peut apporter une contribution importante à la caractérisation de la tradition en question.
\end{abstract}

\begin{abstract}
Series of problems in a Hungarian tradition of mathematics teaching in the 20th century. In this paper, we discuss the interest of studying "series of problems" in order to better characterize a Hungarian tradition of mathematics teaching in the 20th century. In the first part, we summarize its historical context as well as the shared ideas on mathematics and its teaching underlying this tradition. In the second part, we study in more detail the text of one member working in this tradition, namely an excerpt of Rózsa Péter's Playing with Infinity, the study serving as model for the subsequent analyses. In the third part, we present several kinds of historical sources related to the reform of mathematics teaching directed by Tamás Varga in the 1960's and 1970's, given that this reform represents an important stage in the aforementioned "Hungarian tradition". We will attempt to show that the active reflection on the ordering of problems plays a crucial role in the structure of these various documents, many of which are written in a narrative and quasi literary form. We indicate, furthermore, that the study of the "series of problems" contained in these texts can bring much to the characterization of the aforesaid tradition.
\end{abstract}

\footnotetext{
a e-mail : katalin.gosztonyi@gmail.com

${ }^{1}$ Je remercie Francesca Aceto, Alain, Bernard, Aurélien Berra et Jeanne Peiffer pour leur relecture et leurs commentaires qui m'ont beaucoup aidée à améliorer cet article.

This is an Open Access article distributed under the terms of the Creative Commons Attribution License 4.0, which permits unrestricted use, distribution, and reproduction in any medium, provided the original work is properly cited.
} 


\section{SHS Web of Conferences}

\section{Introduction}

La recherche présentée dans cet article s'inscrit dans un projet de thèse aux frontières de l'histoire et de la didactique des mathématiques, dont le titre provisoire est « Traditions et réformes dans l'enseignement des mathématiques à l'époque des «mathématiques modernes » : le cas de la Hongrie et de la France ». Dans la thèse, nous adoptons une approche comparatiste et nous cherchons d'une part à identifier les caractéristiques communes aux réformes française et hongroise, provenant des discours internationaux de l'époque (des décennies 1960 et 1970) ; d'autre part, nous essayons de montrer qu'il y a des différences importantes entre ces deux réformes, remontant à des traditions particulières aux deux pays. Dans le cadre du projet historique interdisciplinaire « séries de problèmes », nous nous intéressons à une caractéristique importante de la tradition hongroise en question : notamment au rôle que les problèmes consciemment mis en ordre jouent dans une pratique pédagogique complexe, à la logique de cette mise en série des problèmes, enfin aux caractéristiques des textes écrits qui reflètent cette pratique et qui pour cette raison peuvent être qualifiés ici de « séries de problèmes ».

Contrairement à la réforme française, largement étudiée par les historiens de l'enseignement des mathématiques (par ex. d'Enfert \& Kahn 2011, Gispert 2008), la réforme hongroise mise en place par Tamás Varga et ses collègues a jusqu'ici était très peu étudiée - en dépit de la valeur et de la pertinence que la communauté hongroise des enseignants de mathématiques lui accorde généralement ${ }^{2}$. La même chose vaut de façon plus générale pour la tradition d'enseignement dans laquelle cette réforme s'inscrit : elle est réputée en Hongrie et au niveau international en tant que «typiquement hongroise », mais il en manque des analyses historiques et didactiques ${ }^{3}$. Un des principaux objectifs de notre recherche est de contribuer à la caractérisation de cette tradition.

On souligne souvent que cette « tradition hongroise » est centrée sur les démarches d'investigation, qu'elle vise à «faire découvrir des mathématiques » aux élèves et que la résolution des problèmes y joue un rôle important; mais ces démarches elles-mêmes sont peu décrites ${ }^{4}$. De nombreux représentants de cette tradition déclarent qu'il est important de baser son enseignement sur des séries bien ordonnées de problèmes ; en même temps, dans les colloques et journaux hongrois, on parle avant tout des problèmes individuels, mais on rend rarement compte de leur mise en ordre et des méthodes qui les organisent en processus d'enseignement.

Dans l'enseignement des mathématiques en Hongrie, un grand nombre de recueils de problèmes sont utilisés : certains d'entre eux sont censés être utilisés en y sélectionnant des problèmes individuels et sans que l'ordre des problèmes soit pertinent (comme les collections pour se préparer au baccalauréat, par exemple Gimes 1980). Dans d'autres cas, la mise en série des problèmes a de l'importance et l'ordre devrait être suivi dans l'enseignement : mais ce fait est rarement explicité par les auteurs, et même s'il est indiqué, les raisons et la logique de la mise en série de problèmes sont peu décrites (c'est le cas des recueils de problèmes de certains manuels scolaires par exemple, comme on verra plus loin).

Nous faisons donc l'hypothèse qu'un des éléments principaux qui est nécessaire pour la bonne caractérisation de cette tradition est la prise en compte de «la mise en série des problèmes ».

\footnotetext{
2 Voir par ex. les remémorations dans les actes des Journées Tamás Varga, colloque annuel de l'enseignement des mathématiques en Hongrie : http://mathdid. elte.hu/html/vtn.html (dernière consultation le 14 mai 2015). Par exemple (Scharnitzky \& Török 2002, Pálfalvi 2007).

${ }^{3}$ Cette image commune est bien illustrée, en langue française, par exemple par le $\mathrm{N}^{\circ} 126$ de la revue Tangente (2009) où un dossier était publié sur cette culture mathématique sous le titre « Le miracle hongrois ». Il existe quelques recherches comparatives en didactique des mathématiques qui s'intéressent à l'enseignement des mathématiques contemporain en Hongrie, voir par ex. (Andrews 2003, Andrews \& Hatch 2001).

4 Voir par exemple les «principes pédagogiques» de la colonie de vacances mathématiques « MaMuT» https://sites . google.com/site/mathematicscamp/oktatasi-alapelveink. (Dernière consultation le 4 mai 2015.) Cette colonie de vacances est organisée par les disciples de Lajos Pósa, mathématicien et enseignant, qui est probablement le représentant contemporain le plus reconnu de la «tradition hongroise».
} 
Au lieu de considérer des problèmes individuels, nous nous intéresserons à leur enchaînement, à la structure des séries, et aux techniques par lesquelles une succession de problèmes et de solutions, une dialectique de questions et de réponses s'organise en processus d'invention et de construction d'un savoir mathématique dans la tradition en question.

Nous nous servons d'une approche historique pour répondre aux problèmes soulevés ci-dessus. Dans la première partie, nous essayons avant tout de reconstituer quelques éléments-clés du contexte historique de l'émergence de cette tradition. Nous détaillerons un moment de cette histoire, qui nous paraît remarquable : le discours d'une communauté de mathématiciens qui, à partir des années 1940, se sont intéressés aux questions d'éducation dès cette époque et ont eu une certaine influence sur la réforme ultérieure de Tamás Varga. Un résumé de leur conception des mathématiques et de leurs principes d'enseignement permet de comprendre les choix épistémologiques derrière le projet pédagogique que ces penseurs représentent.

Dans la suite de l'article, nous étudierons quelques textes écrits par les membres de cette communauté. Nous ne traiterons pas directement des recueils de problèmes mentionnés ci-dessus, contenant des énoncés numérotés, mais de quelques autres textes qui prennent plutôt une forme narrative et quasi-littéraire. Nous tenterons de montrer que ces textes narratifs illustrent en effet une pratique d'enseignement basée sur des séries de problèmes consciemment mise en ordre, et qu'ils permettent de mieux comprendre la logique et les raisons pour organiser les problèmes en série.

Dans la deuxième partie, l'analyse d'un extrait de Jeux avec l'infini, livre de vulgarisation de Rózsa Péter, une mathématicienne de cette communauté, nous servira de premier exemple d'analyse de texte. Nous montrerons l'intérêt d'y chercher à identifier une série de problèmes pour éclaircir la structure du texte. Ce premier exemple servira en quelque sorte de modèle pour l'analyse ultérieure des documents de la réforme de Varga, présentés dans la troisième partie. Nous présenterons différents types de textes liés à la réforme de Varga et nous discuterons l'intérêt de chercher à les interpréter en tant que séries de problèmes. A la fin de l'article, nous illustrerons sur un exemple très simple de recueil de problèmes, issu d'un manuel de collège de l'équipe de Varga, l'apport des analyses antérieures dans l'interprétation de ce texte.

\section{Le contexte historique - Les Mathématiques et leur enseignement en Hongrie au $20^{\mathrm{e}}$ siècle}

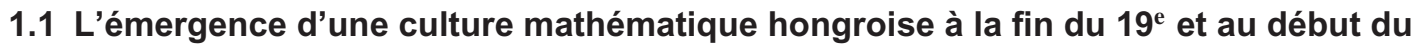 $20^{\mathrm{e}}$ siècle}

Avant les dernières décennies du $19^{\mathrm{e}}$ siècle, il y avait très peu de recherches mathématiques en Hongrie. Les seuls mathématiciens significatifs de l'époque sont les deux Bolyais, Farkas et János. A partir du tournant du siècle par contre, une vie mathématique riche et animée émerge dans le pays : durant le $20^{\mathrm{e}}$ siècle, un nombre étonnamment grand de mathématiciens importants a été éduqué en Hongrie.

Les raisons de cette évolution soudaine ne sont pas entièrement claires, bien qu'elles soient discutées par plusieurs travaux (par ex. Hersch, John \& Steiner 1993, Császár 2005, Frank 2012). Les changements économiques et sociaux de l'époque, l'industrialisation rapide et la formation d'une nouvelle classe moyenne jouent certainement un rôle, ainsi que les réformes de l'éducation des dernières décennies du $19^{e}$ siècle. En ce qui concerne l'enseignement des mathématiques, ce sont des mathématiciens de premier ordre qui travaillent sur les programmes et les manuels du secondaire au tournant du siècle. Pour sélectionner et motiver les élèves doués en mathématiques, différentes compétitions mathématiques sont fondées, et le journal KÖMAL propose aux lycéens des problèmes à résoudre tous les mois. 
Ces compétitions et le journal - qui existe encore aujourd'hui ${ }^{5}$ - jettent les bases d'une tradition de résolution de problèmes dans la culture mathématique hongroise dès la fin du $19^{\mathrm{e}}$ siècle. $^{6}$

\subsection{Le cercle de Karácsony et les mathématiciens - conceptions des mathématiques et de son enseignement au milieu du $20^{\mathrm{e}}$ siècle}

Sándor Karácsony (1891-1952) est un pasteur calviniste, pédagogue et psychologue, qui a eu une influence importante dans les décennies 1930 et 1940. Par contre, à l'époque communiste, il était considéré comme «politiquement problématique » au point que même ses disciples font très peu de références explicites à lui. Dans les années 1940, un cercle d'intellectuels s'est formé autour de lui pour discuter des questions d'éducation. Plusieurs mathématiciens importants participent à ce cercle : László Kalmár (1905-1976), Rózsa Péter (1905-1077), János Surányi (1918-2006) ainsi que le jeune professeur de mathématiques, Tamás Varga (1919-1987), futur directeur de la réforme du primaire. ${ }^{7}$ Ces mathématiciens se sont retrouvés plus tard, à partir des années 1950, dans l'institut de recherche mathématique fondé par Alfréd Rényi (1921-1970). Ils gardaient tous un intérêt pour les questions d'enseignement et ont eu plus tard une influence plus ou moins importante sur la réforme de Varga. Leurs idées sur les mathématiques et sur leur enseignement s'expriment dans leurs correspondances, dans quelques communications sur l'enseignement, ainsi que dans leurs œuvres de vulgarisation qui restent populaires jusqu'à aujourd'hui. Outre le livre Jeux avec l'infini de Rózsa Péter, mentionnons l'exemple de Rényi qui a écrit des pastiches dans le style de Platon, Galilée et Pascal.

Par ailleurs, le futur historien des mathématiques Árpád Szabó (1913-2001) et le futur philosophe des sciences et des mathématiques Imre Lakatos (1922-1974) étaient aussi en contact avec Karácsony et les mathématiciens mentionnés; et dans les années 1950, ils travaillaient tous les deux dans l'institut de Rényi. Plusieurs articles récents ont insisté sur les diverses influences probables entre toutes ces personnes (Gurka 2001, Máté 2006, 2008, Szabó 2013).

Enfin, mentionnons le nom de György Pólya ${ }^{8}$ (1887-1985) : faisant sa carrière à l'étranger, il avait peu de contact direct avec ce groupe, mais il avait fait ses études dans le même milieu et son œuvre était une référence très importante pour la réforme de Varga.

Une analyse des divers textes de ces penseurs nous a permis d'identifier les traits principaux de leur conception des mathématiques et de son enseignement, dont je propose le bref résumé suivant ${ }^{9}$ :

1. Les mathématiques ne sont pas considérées comme statiques et éternelles mais plutôt comme une création de l'esprit humain, comme quelque chose qui change et évolue sans cesse. Les élèves doivent être accompagnés selon le même processus évolutif de création.

\footnotetext{
${ }^{5}$ KÖMAL est disponible en anglais : http://www.komal .hu/info/bemutatkozas.e.shtml

${ }^{6}$ En France, un projet de recherche récent, le projet Cirmath (Circulation des mathématiques dans et par les journaux : histoire, territoires et publics) vise à étudier les périodiques mathématiques comme sources principales de la circulation des mathématiques entre différents territoires et milieux à l'époque moderne. Voir le blog du projet : http://cirmath.hypotheses.org/a-propos (dernière consultation le 14 mai 2015) ainsi que le billet de J. Peiffer http://problemata.hypotheses.org/389.

${ }^{7}$ Kontra, un disciple de Karácsony mentionne le nom de Kalmár, Péter et Varga comme participants réguliers du cercle (Kontra 1992). Quant à la participation de J. Surányi, c'est son fils, László Surányi, qui me l'a confirmée lors d'une discussion personnelle. Surányi était d'ailleurs le rédacteur en chef de KÖMAL qu'il a relancé après 1945 et il était le directeur de l'équipe qui a élaboré les programmes de la première classe spécialisée en mathématiques. Sa contribution est donc significative pour l'enseignement des mathématiques, mais concerne plutôt le niveau du lycée et ne fait donc pas l'objet de notre recherche actuelle.

${ }^{8}$ Le mathématicien György ou George Pólya est renommé avant tout pour son livre How to solve it (Comment poser et résoudre un problème) sur l'enseignement de la résolution des problèmes en mathématiques, qu'il a publié en 1945 aux États-Unis. Il a publié aussi un recueil de problèmes avec Gábor Szegô, et plusieurs œuvres plus théoriques sur le sujet.

9 J'ai présenté cette analyse en détail au colloque du projet « Mathematical Cultures » à Londres en 2012 (Gosztonyi à paraître).
} 
2. La source des mathématiques est l'intuition et l'expérience (non limitée aux observations physiques réelles). Il importe de développer l'intuition à l'aide d'expériences multiples, riches et diverses, à tous les niveaux de l'éducation.

3. L'activité mathématique est essentiellement dialogique, il s'agit d'une série de questions, de problèmes et de tentatives pour y répondre. L'enseignement des mathématiques est une activité conjointe de l'élève et de l'enseignant.

4. Tout formalisme inutile est découragé, l'utilisation d'un langage formel ne doit être introduite qu'après une préparation appropriée.

5. Le but de l'enseignement des mathématiques n'est pas de transmettre de manière irréfléchie des recettes de calculs, mais plutôt de fournir une initiation au processus de la création mathématique, et par conséquent d'éduquer les gens à réfléchir.

6. Le processus de la création mathématique est en relation étroite avec le jeu, et dans cet aspect ludique, c'est la nature artistique des mathématiques qui se manifeste.

\subsection{Le mouvement de réforme dirigé par Tamás Varga entre 1963 et 1970}

Tamás Varga travaillait à l'Institut Pédagogique National depuis la fin des années 1940. Une série de conférences de Zoltán P. Dienes ${ }^{10}$ en 1960 et un colloque international de l'UNESCO sur l'enseignement des mathématiques organisé à Budapest en 1962 l'ont inspiré et poussé à commencer des expérimentations à l'école primaire. Le cercle des participants était progressivement élargi et, au début des années 1970, après l'examen de plusieurs expérimentations se déroulant dans le pays, une commission ministérielle a choisi celle de Varga comme base du nouveau programme prévu pour les premières huit années de la scolarisation. Il a été introduit en 1978.

Ce n'est pas l'objet ici de rentrer dans les détails de la réforme. J'insisterai seulement sur ce qui fait sa spécificité dans le paysage international de l'époque. En effet, cette réforme s'inscrit clairement dans le mouvement international de l'époque «mathématiques modernes » (ou «new math » en anglais) des décennies 1960 et $1970^{11}$. Varga et ses collègues ont activement participé aux activités des divers organismes internationaux (comme le CIEAEM, le CIEM ou l'UNESCO par exemple) et ont collaboré avec des chercheurs et enseignants de plusieurs pays. ${ }^{12}$ La réforme hongroise suit plusieurs prétentions partagées au niveau international : elle vise à introduire les domaines nouvelles de mathématiques dans l'enseignement (comme la théorie des ensembles, la logique, la topologie etc.) et à présenter des mathématiques comme une science cohérente ; elle s'appuie sur les résultats de la psychologie de l'apprentissage ; elle cherche à utiliser des méthodes pédagogiques modernes, surtout à l'enseignement primaire.

En même temps, il importe de souligner des caractéristiques particulières qui lient cette réforme à la tradition hongroise représentée, parmi d'autres, par les mathématiciens du cercle de Karácsony. Comme on verra plus loin, et contrairement aux nombreuses autres réformes de l'époque (notamment en France), Varga refuse l'utilisation excessive de l'abstraction et du langage formel, et insiste plutôt sur le rôle de l'intuition ; il met l'accent sur la nature évolutive et dialogique des mathématiques, sur les processus d'invention guidés par des séries de problèmes, et sur l'aspect ludique et artistique des mathématiques.

\footnotetext{
${ }^{10}$ Chercheur sur l'enseignement des mathématiques d'origine hongroise, mais vivant surtout dans différents pays anglo-saxons. Les outils et jeux développés par Dienes ont eu une grande influence sur l'enseignement des mathématiques dans le monde entier pendant les décennies 1960 et 1970.

${ }^{11}$ Ce mouvement, partant des États-Unis et d'Europe de l'Ouest, a touché un grand nombre de pays du monde entier. Les discours internationaux intenses, de nature politique, scientifique et pédagogique, menés au sein de plusieurs organismes internationaux, ont assuré une certaine cohérence entre les réformes de l'enseignement des mathématiques de l'époque, malgré les différences nationales. La France était un des pays centraux de ce mouvement. Pour plus de détails voir par ex. (Gispert 2011, Kilpatrick 2012).

12 Dans sa thèse, Varga détaille la nature de ses collaborations internationales (Varga 1975). Il publie ensemble avec des collègues canadiens, belges, français entre autres (par ex. Servais \& Varga 1971, Glaymann \&Varga 1973, Varga 1982).
} 


\section{Un premier exemple : séries de problèmes dans Jeux avec l'infini de Rózsa Péter}

Rózsa Péter (1905-1977) est une mathématicienne connue surtout pour ses recherches sur les fonctions récursives. Enseignante du secondaire avant la deuxième guerre mondiale, elle a gardé durant toute sa vie un intérêt pour les questions d'éducation. Elle s'est aussi intéressée aux arts et a souvent défendu les liens entre arts et mathématiques. Son ouvrage le plus connu est sans aucun doute Jeux avec l'infini, un livre qu'elle a écrit pendant la guerre et adressé avant tout aux littéraires, aux «non-mathématiciens », pour « offrir à des esprits curieux une vue d'ensemble de notre discipline », pour leur faire sentir « la joie de la découverte » (Péter 1977 p. 9). Le livre est très populaire, il est régulièrement réédité en Hongrie et a été traduit dans douze langues (dont le français en 1977). ${ }^{13}$ Il semble aussi avoir eu une influence sur l'enseignement ${ }^{14}$. Le premier livre du maître par exemple, qui accompagne le programme réformateur de T. Varga en 1978, se réfère explicitement à Jeux avec l'infini pour ce qui concerne certaines questions mathématiques et didactiques.

Dans ce livre, l'auteur mène ses lecteurs des notions mathématiques les plus simples aux résultats contemporains les plus abstraits, comme les théorèmes de Church ou de Gödel. Elle emploie pour cela un style littéraire riche en références historiques et culturelles, presque sans employer aucune formule. Une analyse fine permet pourtant de dévoiler derrière le texte continu, facile à lire, une structure complexe et sophistiquée, fondée sur un enchaînement de questions et de problèmes.

Notre analyse concerne les chapitres 4 et 5 du livre ${ }^{15}$. Le chapitre 4 commence par un court discours sur les nombres « amis » et les nombres «parfaits », ce qui établit la transition avec le chapitre précédent (portant sur les questions de divisibilité des nombres naturels), et amène rapidement à des questions simples mais non résolues de la théorie des nombres. L'auteur en profite pour insister sur l'importance du pouvoir créatif et de la curiosité humaine dans la recherche mathématique.

L'homme, pour sa propre commodité, a créé la suite des nombres naturels, qui permet de compter les objets et de faire un certain nombre d'opérations. Seulement, bien que l'ayant créée, l'homme n'a pas la maîtrise de cette suite : elle possède désormais ses propres règles, que l'homme n'avait absolument pas prévues. Tel un apprenti sorcier, il contemple les yeux éblouis, les djinns qu'il a libérés. Le mathématicien crée, à partir de rien, un univers nouveau. ${ }^{16}$ Mais les régularités mystérieuses et inattendues de cet univers "captivent " l'homme, au sens étymologique du terme, et, de créateur, il se transforme en chercheur, il s'adonne à la recherche des secrets de sa création devenue indépendante de lui (p. 41).

La suite du chapitre en donne un exemple, décrivant une expérience de classe :

Recherche séduisante qui n'exige pour ainsi dire aucune formation, mais seulement une certaine dose de curiosité. C'est ainsi qu'un jour une de mes élèves de sixième me dit : "Je me suis aperçue, il y a longtemps déjà, qu'en additionnant des nombres qui se suivent, si je m'arrête à un nombre impair, par exemple à $7, j$ 'obtiens un nombre qui est égal au produit de ce nombre et de son milieu; par exemple, le milieu de 7 est 4 (par « milieu » elle voulait dire, de toute évidence, le chiffre qui se trouve au milieu de la séquence allant de 1 à $7: 1,2,3,4,5,6,7)$, et 7×4=28. Quant à la somme des nombres allant de $1 \grave{a} 7,1+2+3+4+5+6+7$ fait également 28 . Je sais que c'est toujours comme ça, mais je ne sais pas pourquoi.»

\footnotetext{
13 Une nouvelle édition française du livre est parue en 2014, qui est donc facilement accessible aux lecteurs intéressés.

14 Elle est aussi l'auteur, avec le mathématicien Tibor Gallai et autres, d'une série de manuels scolaires du lycée, qui était utilisée en Hongrie pendant des années 1950. (Gallai \& Péter 1949).

15 Cette analyse est présentée plus en détails dans (Gosztonyi 2014).

16 «L'apprenti sorcier » réfère à un poème de Goethe de même titre. La phrase suivante est une paraphrase d'une célèbre citation de János Bolyai : «J'ai créé, à partir de rien, un univers nouveau » écrit-il à son père en lui présentant son invention, la géométrie hyperbolique (Lettre du 3 novembre 1923, citée par exemple dans Prékopa et al. 2004).
} 


\section{"C'est une progression arithmétique, me dis-je, mais comment l'expliquer au niveau de la sixième ? » Pour commencer, je soumis le problème à la classe : «Suzy a un problème intéressant... » (ibid.)}

C'est ainsi que commence un long processus de recherche : les élèves trouvent rapidement la réponse à la question (l'auteur-enseignant remarque que «Jamais je n'aurais su expliquer la solution aussi bien qu'elle » [elle : Ève,uneautreélèvedelaclasse] (p. 42), mais la question, ainsi que la méthode utilisée pour y répondre, amène à d'autres questions, à des problèmes analogues, ou bien très différents en apparence mais qui ont un point commun avec le problème original. À la fin de ce processus, dans le chapitre 5, on arrive à une formule, qui est censée résumer une variété de problèmes différents :

La formule ci-dessus [...] ne signifie rien de précis en elle-même, et chacun peut s'en servir pour exprimer son expérience individuelle. Pour les uns, elle exprimera le nombre des diagonales d'un polygone de $n$ côtés, pour les autres, le nombre de façons de mettre n élèves « en rangs par deux ». La formule exprime avant tout notre satisfaction de voir toute cette diversité ramenée à une unité. (p. 53.)

Notre schéma d'analyse (Fig. 1) essaye de présenter la structure de cet extrait comme une série de problèmes, et de rendre compte des «étapes » de cette série : des problèmes et des solutions, ou des questions et des réponses, et des liens qui enchaînent ces étapes. Nous considérons ce schéma comme une version intermédiaire, qui laisse plusieurs problèmes d'analyse ouverts, mais qui permet à la fois de mettre en lumière certaines caractéristiques du texte de Péter, et de le comparer à d'autres textes.

Une des difficultés est le découpage du texte, l'identification des étapes : car il est en forme continue, littéraire, il n'y a pas d'énoncés numérotés par exemple. Il faut donc trouver des marqueurs linguistiques qui permettent de distinguer des étapes. De tels marqueurs peuvent être par exemple les formules comme «Il est évident que ...", qui cachent chaque fois une question, traitée dans le paragraphe qui suit. Mais l'identification des étapes reste pour l'instant, dans de nombreux cas, intuitive, sans critères d'identification systématiques. ${ }^{17}$ Une définition plus stricte de critères nécessiterait une analyse sur une plus grande échelle (sur le livre entier et éventuellement sur d'autres documents) ${ }^{18}$.

Une deuxième difficulté concerne la catégorisation de ces étapes et la terminologie qu' on y utilise ${ }^{19}$. Dans la première version de la présente analyse, nous avons interprété chaque étape en tant que « problème ». Mais ce seul terme est apparu insuffisant, car ces «problèmes » sont de natures très différentes : les « problèmes » consistant à trouver un algorithme pour additionner des nombres successifs ou calculer la surface d'un triangle se situent à un niveau différent des «problèmes » qui demandent comment utiliser notre résultat ou réinvestir la méthode de notre solution dans d'autres champs mathématiques. À ce stade de l'analyse, nous distinguons deux niveaux : celui des « problèmes » de la première catégorie et celui de «questions de recherche » pour la deuxième. Cette distinction nous paraît fructueuse pour plusieurs raisons que nous exposerons plus loin.

Une troisième difficulté concerne la nature des flèches : elles représentent des transitions d'une étape à l'autre, mais à nouveau, il faut définir à terme plus systématiquement ces liens et décrire leur nature. En général donc, l'analyse du texte reste intuitive sur plusieurs points : une analyse à une plus grande échelle serait certainement nécessaire pour stabiliser les critères.

En même temps, malgré les difficultés et limites présentées ci-dessus, la schématisation du texte de Rózsa Péter nous paraît intéressante pour plusieurs raisons. Elle permet avant tout de dévoiler, derrière

\footnotetext{
${ }^{17}$ L'extrait a été étudié à plusieurs reprises avec des enseignants stagiaires de la formation continue « Problèmes et énigmes au carrefour des cultures ». (http://problemata.hypotheses.org/172, http://problemata.hypotheses.org/398) Malgré la relative intuitivité de l'analyse, les participants se trouvaient à peu près d'accord sur l'identification des étapes. Par contre, au séminaire de recherche du 11 octobre 2013 (http: //problemata . hypotheses . org/217), un débat s'est ouvert sur les questions d'identification et plusieurs modifications étaient proposées.

18 Le découpage du texte est un problème partagé par plusieurs membres du projet, et même dans le cas des textes qui comprennent des énoncés clairement distinguables : voir par exemple dans ce volume l'article de M. Moyon qui suggère une division des textes à l'aide des figures qui appartiennent chaque fois à un ensemble d'énoncés.

19 À nouveau une question qui préoccupe plusieurs membres du projet (voir Sects. 1.5 et 1.6 de l'introduction générale).
} 


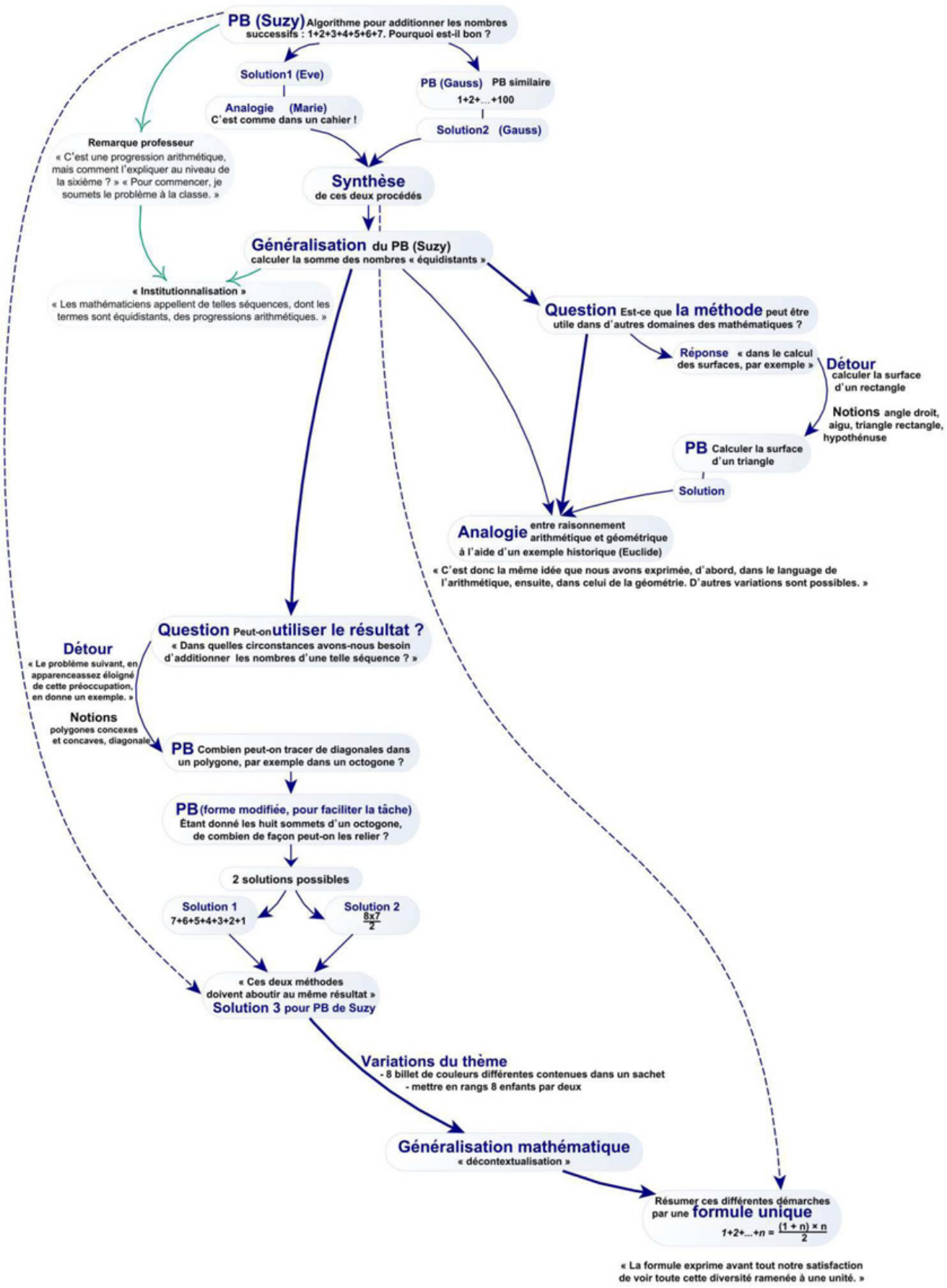

Figure 1. Schéma d'analyse du texte de R. Péter. 
un texte simple et transparent en première apparence, une structure complexe et sophistiquée qui prend la forme d'un jeu dialectique de questions, de tentatives de réponses et de nouvelles questions qui émergent de ces tentatives. On voit une démarche pédagogique fondée sur des problèmes, mais il ne s'agit pas de problèmes mathématiques isolés qu'on abandonne après la solution, bien au contraire ; ce sont des questions de recherche autour de problèmes et de leurs solutions : des essais de réinvestissement des méthodes utilisées ou des résultats, la comparaison des différentes solutions d'un même problème, la dialectique établie entre différents domaines mathématiques à l'aide de l'analogie, qui font avancer les lecteurs/élèves, guidés par l'auteur/enseignant, dans le processus de construction et de développement des connaissances mathématiques.

La distinction entre «problèmes » et «questions de recherche » permet aussi de découvrir une analogie entre le texte de Rózsa Péter et le livre How to solve it (Comment poser et résoudre un problème) de G. Pólya ${ }^{20}$. Au début de son livre, Pólya propose une liste de questions qui servent à aider les lecteurs quand ils veulent résoudre des problèmes. Par exemple : «Voici un problème qui se rattache au vôtre et que vous avez déjà résolu. Pourriez-vous vous en servir ? Pourriez-vous vous servir de son résultat? Pourriez-vous vous servir de sa méthode ? » ou « Pourriez-vous imaginer un problème qui s'y rattache et qui soit plus accessible ? Un problème plus général ? Un problème plus particulier ? Un problème analogue ? » (Pólya 1965, après la table des matières). D’une part, on voit que ce sont des questions très similaires qui guident la démarche de Rózsa Péter ; de l'autre, cette comparaison attire l'attention sur le fait que la majorité des questions proposées par Pólya renvoient à un contexte de problèmes déjà résolus, ou propose de modifier ou faire varier le problème original (donc créer une série de problèmes...). En fait, cette approche permet de ne plus voir dans les questions de Pólya un questionnaire pour résoudre des problèmes isolés, mais un guide pour construire progressivement un espace organisé de problèmes qui permet d'y situer les nouveaux problèmes émergeants.

L'extrait de Péter illustre bien les principes de la communauté hongroise, que nous avons détaillés plus haut (cf. Sect. 1.2). Les mathématiques apparaissent comme une création humaine en plein développement : leur aspect créatif et ludique est explicitement souligné. Le professeur n'apparaît pas, dans cette démarche pédagogique, comme l'autorité indiscutable, l'unique source d'informations, mais comme un guide expérimenté dans un processus de recherche, activité conjointe des élèves et de l'enseignant. L'utilisation du langage formel des mathématiques mérite également une attention particulière : les démonstrations et les raisonnements ne sont pas présentés sous forme abstraite et générale, mais toujours sur des exemples concrets. Toutefois, ces exemples sont toujours génériques, les raisonnements permettent de comprendre des idées, des théorèmes généraux. Le langage formel n'intervient qu'à la fin du processus : il aide à décontextualiser et à unifier divers problèmes et solutions analogues.

Enfin, nous attirons l'attention sur une question rhétorique, la forme semi-dialoguée de l'extrait. Une partie du texte prend la forme d'un dialogue et distingue clairement les voix des élèves et celle de l'enseignant; dans d'autres parties, on n'entend qu'un narrateur qui masque les voix dialoguant entre elles. On va retrouver cette solution rhétorique dans certains manuels scolaires des années 1970.

\section{Le corpus didactique : documents de la réforme de l'enseignement des mathématiques}

Dans cette partie de l'étude, nous nous intéressons aux différents documents écrits liés à la réforme de Varga. En fait notre recherche, partiellement didactique, s'intéresse aux pratiques d'enseignements qui sont bien plus complexes que ce qu'en reflète la documentation écrite disponible. Mais cette

\footnotetext{
${ }^{20}$ Les deux livres datent de la même période et ont été rédigés pendant la deuxième guerre mondiale, l'un aux États-Unis, l'autre en Hongrie. On peut supposer qu'il n'y avait pas de communication directe entre les deux auteurs.
} 
étude pose un problème méthodologique important, notamment le manque des vidéos ou d'autres moyens d'observation des classes des années 1970. Notre recherche n'envisage donc pas d'étudier directement les pratiques réelles dans les classes, mais essaye, dans une première étape, de reconstruire l'enseignement visé par les concepteurs des réformes et par les auteurs de manuels à partir de documents écrits. Nous considérons ces documents comme des textes historiques dont une analyse textuelle soigneuse paraît nécessaire pour contribuer à un travail de reconstruction des pratiques d'enseignement. Nous verrons par ailleurs que cette distinction des sources écrites aura l'avantage pour le long terme de pouvoir identifier des éléments préparatoires, consistant surtout en une mise en ordre des problèmes, et des éléments improvisés, principalement en forme dialogique, au sein d'une pratique pédagogique complexe (cf. Sect. 4).

Nos principales sources sont les programmes et les instructions, les fiches de travail, les manuels scolaires, les livres du professeur. Nous concentrons notre étude sur les premiers huit ans de l'éducation (ce sont les niveaux scolaires concernés par la réforme de Varga, correspondant à peu près à l'école primaire et au collège français). Il faut préciser en outre que, dans l'époque concernée en Hongrie, contrairement à la France, il n'existait qu'une seule série de manuels scolaires obligatoire. Nous étudierons donc les textes pédagogiques qui accompagnaient le programme de Varga en 1978 et qui étaient rédigés par la même équipe.

Il s'agit de textes de différentes natures et l'intérêt d'y étudier les séries de problèmes apparaît différemment dans le cas de chacun des types. Dans la suite, nous résumerons quelques aspects de l'analyse de ces documents, et nous tenterons de montrer chaque fois l'intérêt d'y chercher à étudier des « séries de problèmes ».

Une remarque préalable concernant la terminologie s'impose : bien que le mot «problème » apparaisse dans les textes hongrois plus théoriques, les documents d'enseignement l'utilisent très rarement, on y parle surtout de «tâches ». Nous reprenons cette terminologie dans la suite de l'article, sans vraiment questionner pour le moment le lien ou la distinction entre « tâches » et "problèmes », tout en sachant que ce travail sur la terminologie devra être fait dans le futur. Nous remarquons simplement que le mot «tâche » semble être plus général, indiquant toutes sortes de questions qui demandent une réponse ou une activité ; le mot "problème » semble désigner des questions dont la solution pose une difficulté, où la méthode de solution n'est pas connu au préalable et doit être cherchée par les élèves. Par contre, on applique parfois le terme « exercice » qui désigne l'application d'une connaissance ou méthode donnée.

\subsection{Le programme}

Avant de présenter des manuels et des livres de maîtres, nous soulignons une caractéristique du programme de Varga. À l'époque des mathématiques modernes, de nouveaux contenus sont introduits dans les curricula à travers les réformes menées dans différents pays. De plus, on insiste généralement sur la cohérence de la matière et on commence à parler de " mathématiques » - ou même de « la mathématique » en France - au lieu de « calcul » ou de « calcul et mesure ». Mais l'établissement de cette cohérence et la structure des programmes ne suivent pas la même logique : quand les programmes français adoptent un ordre plutôt hiérarchique et linéaire, fondé sur la théorie des ensembles, le programme hongrois de Varga développe parallèlement cinq grands domaines mathématiques (ensembles-logique ; arithmétique-algèbre ; relations-fonctions-séries ; géométriemesure ; combinatoire-probabilités-statistique) et vise à établir une dialectique riche entre eux. Par conséquent, l'enseignement correspondant au programme hongrois ne peut pas simplement mettre un grand thème après l'autre, il est obligé de les développer plus ou moins parallèlement pour créer une réelle dialectique entre les différents domaines. 


\subsection{Les fiches de travail et livres du maître pour le primaire : une stratégie d'enseignement par mise en série des problèmes}

Au niveau de l'école primaire, on utilise, à l'époque, surtout des fiches de travail plutôt que des manuels scolaires. Les fiches elles-mêmes peuvent être interprétées comme des séries de problèmes. Elles sont numérotées et chaque fiche comprend plusieurs tâches, qui sont parfois en lien apparent l'une avec l'autre, mais dans d'autres cas, celles-ci concernent des thèmes différents et semblent être indépendantes. En même temps, les instructions du programme et les livres du maître soulignent que les fiches doivent être considérées seulement comme un outil parmi d'autres dans un processus d'enseignement : elles doivent alterner avec des activités au tableau, des activités manipulatoires avec différents matériels etc. Les livres du maître en proposent de nombreux exemples. Il paraît donc plus fructueux d'essayer d'étudier, au lieu des seules fiches de travail, les processus proposés par les livres du maître, dans leur intégralité. Cette étude est loin d'être évidente, car les livres du maître, tout en offrant de nombreuses consignes et suggestions aux lecteurs-enseignants pour construire ces processus, ne présentent pas de processus réellement complet, mais leur laissent une grande liberté et responsabilité. Pour mieux comprendre cette difficulté, il faut avant tout décrire la structure de ces livres, et étudier les consignes d'utilisation ${ }^{21}$.

La première partie, la plus importante du livre de la première année, consiste en des explications thématiques : la description mathématique et didactique du contenu du programme, dans l'ordre des cinq grands domaines. Il s'agit d'un texte continu et peu structuré qui comporte aussi des propositions de tâches : on peut voir un exemple en annexe I, première image ; les tâches sont marquées par un cercle noir, les « variations » d'une tâche par un cercle blanc. L'introduction du livre précise que les tâches présentées sont ordonnées à l'intérieur d'un thème (donc cet ordre peut être suivi dans l'enseignement), mais elle laisse toutefois ouverte la possibilité de modifier l'ordre, encourage à faire une sélection des tâches et à créer de nouvelles variantes. Les descriptions des tâches donnent souvent des indications concernant les possibilités de leur enchaînement avec d'autres tâches.

En même temps, le livre insiste sur l'importance de présenter des thèmes parallèlement, et de créer des points d'intersection entre eux. Pour faciliter ce travail, une version possible (mais pas obligatoire) du développement annuel est présentée dans une deuxième partie : un tableau décrit l'évolution des thèmes parallèles semaine par semaine, ensuite, une proposition du développement annuel est présentée heure par heure. Pour les tâches, ces propositions renvoient aux tâches présentées dans la première partie thématique et aux fiches de travail. Le reste du livre décrit quelques exemples de séances et donne des instructions pour les fiches de travail. Un nouveau tableau d'évolution des thèmes, plus court et schématique, se trouve à la fin du livre (voir annexe I, deuxième image).

Le livre ne présente donc pas de «leçons » dans le sens où une séance serait consacrée à un thème précis, avec des activités d'introduction au début, et une explication d'enseignant à la fin pour institutionnaliser un élément de savoir mathématique, dont l'apprentissage aurait été l'enjeu de la séance. Les séances proposées dans le livre du maître de Varga comportent deux ou trois activités courtes autour de problèmes qui sont parfois connexes, parfois liées à différents thèmes, et qui ne sont que très rarement suivies par un discours du maître. Les liens complexes entre ces problèmes doivent être construits sur le long terme : le livre insiste souvent sur l'importance de réfléchir en termes de processus d'enseignements longs pour permettre aux élèves de construire leurs propres connaissances à travers une grande variété d'activités et d'expériences. Au lieu d'une transmission directe et immédiate des savoirs mathématiques, l'idée est de laisser aux idées beaucoup de temps pour mûrir : dans certains cas même plusieurs années.

Ces caractéristiques structurelles montrent une réflexion approfondie sur l'enseignement par problèmes, et sur leur mise en ordre. Les unités principales de l'enseignement sont, chez Varga,

\footnotetext{
${ }^{21}$ Nous traitons ici le livre du maître de la première année du primaire (C. Neményi et al. 1978) : la structure des livres des autres niveaux est un peu différente, mais les problèmes d'analyse discutés ici restent similaires.
} 
des problèmes, mais la résolution d'un problème n'amène pas directement à un morceau de savoir institutionnalisé. C'est à travers l'expérience de la résolution d'une grande diversité de problèmes, au cours d'un processus long, que les connaissances ainsi émergentes amènent à une généralisation et à une formalisation lente du savoir institutionnel. Ce qui permet la création de ces processus, c'est la mise en ordre complexe et soigneuse des problèmes, suggérée dans le livre par des indications sur leur enchaînement et par le grand nombre des tableaux de développement. Les variations systématiques des différents aspects des problèmes, la diversité des habillages, le jeu sur la distance temporelle, les liens divers entre les problèmes des différents thèmes sont entre autres les outils, qui permettent d'organiser des problèmes individuels en un processus évolutif conduisant à la construction du savoir mathématique.

Il s'agit donc bien d'un processus d'enseignement basée sur la mise en série des problèmes, et qui peut être illustré par des documents prenant la forme de «séries de problèmes ». Mais les séries présentées dans le livre sont seulement partiellement ordonnées : il faut comparer les séries présentées dans les chapitres thématiques avec les références de tâches indiquées dans le développement annuel pour pouvoir reconstruire des séries complètes. En outre, il faut prendre au sérieux l'instruction du livre qui encourage les enseignants à créer leurs propres tâches et construire leurs propres séries, suivant leur propre envie et créativité, les conditions locales, les spécialités des classes, les capacités et l'intérêt des élèves. Il s'agit d'une liberté de l'utilisateur, mais dont on ne peut pas profiter sans bien comprendre ses limites : des interdépendances thématiques complexes entre les tâches proposées, les possibilités de leur variation et les principes de leur enchaînement. En fait, le chercheur essayant de reconstruire les processus d'enseignement envisagés par les auteurs se retrouve dans une situation assez similaire à celle de l'enseignant censé construire son propre enseignement à partir de ces documents : c'est ici que le croisement des approches historique et didactique devient pertinent.

Nous illustrons les tâches du livre du maître avec l'exemple suivant, qui se trouve dans le chapitre arithmétique, dans le sous-chapitre consacré à l'introduction de la notion de nombre par la mesure, dont une étape est la comparaison des grandeurs :

\begin{abstract}
Devinette avec le matériel cuisenaire ${ }^{22}$
Ce jeu offre une bonne occasion pour pratiquer la comparaison des grandeurs.

On cache une réglette, il faut deviner laquelle. Les élèves peuvent poser des questions, telles que "Est-elle plus grande que la violette?", "plus grande que la bleue?", "plus petite que la rouge?". Ils ont une réglette de chaque couleur devant eux, et peuvent mettre de côté les réglettes qui ne sont plus possibles.

Un exemple: on cache la réglette noire.

«Est-elle plus longue que la jaune? " Oui. » (Ils mettent de côté la blanche, rouge, la vert clair, la violette, la jaune).

«Est-elle plus petite que la noire? » «Non. » (Beaucoup peuvent croire qu'elle doit être plus grande que la noire et mettent de côté la noire aussi, en plus de la vert foncé. On peut revenir à ce problème à la fin du jeu, on peut proposer par exemple de comparer une réglette noire avec une autre noire, et voir si l'une est plus grande que l'autre).

"Est-elle plus petite que la bleue?" "Oui. " (Ceux qui se sont trompé tout à l'heure peuvent maintenant croire avoir trouvé la réponse, et suggérer : c'est la brune qui était cachée. Les autres veulent par contre continuer à poser des questions. Écoutons-les, même si c'était effectivement la brune qui était cachée).

Au début, il y aura probablement beaucoup de questions inutiles. Certains élèves peuvent les récuser: si une réglette est plus grande que la jaune, elle est évidemment plus grande que la rouge. Dans ce cas, on peut introduire une nouvelle règle : si quelqu'un sait la réponse à une question, il peut répondre
\end{abstract}

\footnotetext{
22 Le « matériel cuisenaire » est un ensemble de réglettes colorées de différentes longueurs nommé d'après son inventeur George Cuisenaire. La longueur de chaque réglette est un multiple de la longueur de la plus courte, le cube blanc : le matériel est ainsi conçu pour aider à l'apprentissage des nombres et des opérations. Voir le site http://www . cui senaire.eu/fr/?page=index
} 
à la place du maître. Plus tard, on peut introduire l'objectif de deviner avec le moins de questions possibles. (C. Neményi - Göndöcs et al. 1978, p. 99-100. Ma traduction).

On retrouve dans cet exemple le principe du dialogue - et pas seulement conformément aux règles du jeu. Ce sont les désaccords, les débats entre élèves qui font avancer la réflexion collective. Il est conseillé à l'enseignant de ne pas donner de réponse directe, mais de poser des questions adaptées, ou bien de modifier les règles du jeu pour pousser cette réflexion. De fait, ce seul jeu peut constituer en lui-même une mise en série de problèmes, à travers des répétitions et des variations progressives des règles. Mais cette série ne peut pas être entièrement définie à l'avance, car elle doit dépendre des réactions des élèves et s'y adapter : les consignes du livre sont plutôt des petites idées de réactions aux différentes situations qui peuvent se produire en classe.

Ce jeu en est un parmi beaucoup d'autres qui permettent la familiarisation avec la comparaison des grandeurs. Le livre demande au maître très peu d'explicitations d'un élément de savoir qui devrait être acquis à la fin de l'une ou l'autre activité : les connaissances se développent au fur et à mesure à travers des problèmes qu'on rencontre et qu'on essaye de résoudre. Il y a tout de même quelques points où le livre suggère aux enseignants de s'arrêter et d'expliciter une notion mathématique : dans le cas de l'extrait cité, l'exemple de la réglette noire peut être vu comme tel, car il permet d'amener à une discussion sur la notion d'égalité. En effet, l'extrait est suivi dans le livre d'un nouveau chapitre sur la notion d'égalité. Mais il faut souligner, à nouveau, que l'introduction de cette nouvelle notion, au moins dans l'exemple cité, émerge d'une discussion éventuelle entre élèves et n'est donc pas strictement programmée à l'avance, dans le texte écrit. Le jeu, le problème initial ne crée qu'une occasion pour faire émerger une nouvelle notion, mais c'est à travers des dialogues, des questions et réponses successives des élèves et de l'enseignant que la notion pourrait être formulée.

\title{
3.3 Les manuels scolaires du collège : des textes dialogués
}

Pour le niveau collège (de la $5^{\mathrm{e}}$ à la $8^{\mathrm{e}}$ année d'éducation), des manuels scolaires étaient offerts en plus des fiches de travail et des livres du professeur. Les manuels comportent deux parties : la première présente le contenu du programme, introduit les connaissances nouvelles, la deuxième est un recueil de tâches. La première partie prend souvent une forme rhétorique originale : des dialogues fictifs entre élèves. Voici ce qu'en dit le livre du professeur :

\begin{abstract}
Le manuel présente un traitement possible des thèmes. On peut introduire un problème à travers les tâches présentées. On peut guider les élèves pour susciter dans la classe des discussions, des débats similaires à ceux décrits dans le manuel. Si la classe n'est pas suffisamment active, on peut poser des questions à la place des élèves pour provoquer un débat. Les enfants du livre se trompent souvent, défendent des positions fausses, mais peuvent se convaincre les uns les autres, pour arriver finalement à reconnaître les avis corrects et faux. Ne corrigeons pas les erreurs nous-mêmes, guidons plutôt les élèves, ou laissons-les se guider les uns les autres pour qu'ils reconnaissent et corrigent les erreurs eux-mêmes.

Après la séance, on peut donner la lecture du texte du manuel comme devoir à la maison. (Eglesz et al. 1981, p. 5. Ma traduction).
\end{abstract}

Les dialogues présentés dans les manuels peuvent donc être lus comme des modèles d'enseignement, bien qu'ils fonctionnent aussi comme documents de travail pour les élèves. Un exemple, l'introduction de la notion de distance des ensembles de points, se trouve dans l'annexe II (Eglesz et al. 1979, p. 215-217).

Le chapitre en question est introduit par un problème : mesurer la distance d'un fauteuil à une table. Les élèves du manuel se rendent compte d'un désaccord entre eux sur le résultat, et concluent qu'il faut se mettre d'accord sur la définition de la distance. Après débat, ils acceptent comme distance la longueur du plus long segment entre les deux objets. Ensuite ils sont confrontés à un nouveau 
problème : la distance d'un stylo à une tache. Ils découvrent que la définition acceptée précédemment donne un résultat contradictoire à leur intuition. Dans le cas du troisième problème (la distance d'un point à une droite), la définition précédente ne permet même pas de déterminer une distance entre les deux figures. Il paraît donc souhaitable de modifier la définition : les élèves se mettent d'accord sur une autre définition, qui permet de répondre de façon satisfaisante aux trois problèmes cités (la longueur du plus court segment entre les deux ensembles de points). Ce sera la définition retenue par le manuel.

Les problèmes de cet extrait servent à mettre en contexte une notion mathématique : la distance des ensembles de points. La définition de cette notion n'est pas donnée a priori : elle est créée par un consensus de la communauté, conformément aux intuitions des membres de cette communauté concernant le sens de la notion. La création de cette notion est un processus dialectique où les problèmes jouent le rôle de contre-exemples : ils déstabilisent progressivement la première définition acceptée et amènent à une définition applicable à un niveau plus général. Le processus reste ouvert : la définition retenue paraît satisfaisante, mais n'exclut pas que de nouveaux problèmes se présentent dans le futur, qui nécessiteraient une redéfinition ultérieure de la notion. ${ }^{23}$ Ce processus montre par ailleurs une ressemblance remarquable avec ce qu'on trouve dans Preuves et réfutations d'Imre Lakatos : un jeu dialectique des tentatives de preuve, de définitions et de contre-exemples, présenté aussi dans la forme d'un dialogue entre élèves fictifs d'une classe.

Dans d'autres dialogues issus de ces manuels, on peut identifier davantage de fonctions remplies par les problèmes mis en série : elles permettent de motiver la démonstration mathématique - cf. le cas du théorème de Pythagore où les variations d'un problème amènent à des méthodes de solution radicalement différentes - ou contribuent à justifier un algorithme en créant différents contextes d'interprétation (le cas de la comparaison des fractions). Une étude systématique de ces dialogues serait nécessaire pour faire un catalogue des différentes fonctions que les séries de problèmes peuvent y remplir.

Il est intéressant d'observer la similitude stylistique entre les dialogues des manuels scolaires et le texte de Rózsa Péter. Dans les deux cas, il s'agit de textes quasi continus et peu structurés (avec de nombreuses illustrations dans le cas des manuels). En fait, les manuels présentent aussi des textes semi-dialogués : bien que les dialogues marqués des élèves prennent une place importante, ils sont interrompus par des passages narratifs et des passages où les voix des locuteurs ne sont pas distinguées. C'est le cas, dans l'extrait cité, des problèmes eux-mêmes, alors que dans certains autres extraits les élèves proposent des problèmes les uns aux autres. Si l'on interprète ces textes comme des modèles d'enseignement, ainsi que le suggère le livre du professeur, il est raisonnable de supposer que les passages « anonymes » représentent la voix de l'enseignant. On peut aussi les interpréter comme des passages dont le responsable n'est pas défini à l'avance : si les élèves ne les proposent pas, l'enseignant peut y intervenir en les prenant en charge. Le livre du professeur précise en effet qu'on attend dans les classes des dialogues similaires, mais pas exactement les mêmes, c'est-à-dire que les textes des manuels permettent d'illustrer des types d'interventions attendues des élèves et des types d'interventions que l'enseignant peut faire pour guider et faire avancer la recherche collective. À nouveau, une grande liberté est laissée à l'enseignant qui doit s'adapter aux caractéristiques de sa classe et faire un travail significatif d'improvisation suivant les réactions des élèves.

Pour conclure l'analyse, nous traitons brièvement un autre extrait des manuels de collège, issu cette fois de la deuxième partie qui prend la forme traditionnelle d'un recueil de tâches. L'extrait qui se trouve dans l'annexe III est lié au théorème de Pythagore. Il s'agit des énoncés numérotés : mais, comme on l'a dit dans l'introduction, il n'est pas clair à première vue que les tâches puissent être choisies et résolues

\footnotetext{
23 En effet, lors d'une présentation dans un contexte mathématique universitaire (stage « séries de problèmes » 2014-15, http://problemata.hypotheses.org/398), on m'a rappelé l'insuffisance de cette définition si on veut prendre en compte des ensembles pas continus. Comme ce problème n'apparaît pas dans le contexte du collège, la définition acceptée est suffisante pour ce niveau, mais doit être révisée et généralisée à un niveau ultérieur.
} 
aléatoirement, ou bien si leur l'ordre a une pertinence. Prenons l'exemple des tâches de 124 à 133 : les énoncés se répètent, avec une variation légère entre 126 et 128 , et avec la variation des nombres. Cette liste de tâches pourrait servir à pratiquer l'application du calcul de la distance des points dans un repère, sur un grand nombre d'exemples indépendants. Mais la notion de distance dans un repère n'était pas introduite au préalable dans le manuel.

Si on regarde plus soigneusement les variables, on reconnaît une certaine progressivité entre les tâches : par exemple, distance d'un point de l'origine, avec l'introduction progressive des nombres négatifs ; ensuite, distance entre deux points sur une droite parallèle à un axe, distance entre deux points quelconques, enfin généralisation formelle. Il s'agit donc de problèmes mis en ordre, qui permettent d'élaborer progressivement une méthode de calcul de la distance des points, et amène enfin à une généralisation et une expression formelle de la notion de distance des points dans un repère.

Le processus montre une certaine similitude avec les exemples rencontrés dans les textes narratifs de Péter et de Varga : l'analyse préalable de ces exemples nous aide à reconnaitre, dans la liste des tâches sur la distance, une « série de problèmes », et à comprendre la logique et la visée de cette série.

\section{Conclusion}

Dans cet article, nous avons essayé de présenter l'importance que joue la mise en série de problèmes dans une tradition d'enseignement des mathématiques qui a émergé en Hongrie au $20^{\mathrm{e}}$ siècle.

Nous avons insisté sur la cohérence de cette tradition : les principes résumés dans la première partie et les caractéristiques du texte de Rózsa Péter, étudiés dans la deuxième partie sont en majorité identifiables aussi dans les documents de la réforme de Varga. Les mathématiques y apparaissent en plein développement ; l'activité mathématique se présente comme un processus créatif, basé sur une dialectique de problèmes et de tentatives de solutions. L'enseignement des mathématiques est considéré comme une activité conjointe de l'enseignant et des élèves, qui prend principalement une forme dialogique.

Nous avons identifié plusieurs traits stylistiques communs des textes élaborés dans cette tradition : il s'agit des textes prenant une forme apparemment souple, peu structurée, alternant des passages narratifs et dialogiques et contenant de nombreuses illustrations. Le dialogue en classe, entre élèves et enseignant, est une situation récurrente qu'on retrouve chez R. Péter, dans les livres du maître du primaire et des manuels de collège de Varga ainsi que, dans une forme légèrement différente, dans les œuvres de Rényi, de Lakatos et de Pólya.

Nous nous sommes intéressées aussi à la structure sous-jacente de ces textes, celle là même qui permet de les désigner comme "séries de problèmes ». Les exemples étudiés illustrent comment ces textes sont construits sur un enchaînement complexe et sophistiqué de problèmes, et que cet enchaînement est guidé par certaines questions de recherche. Les séries de problèmes et les questions de recherche apparaissent dans une forme légèrement différente en fonction de l'objectif et du public visé de chaque texte : par exemple, R. Péter les décrit dans une forme principalement narrative, ressemblant presque à un roman, car elle vise à illustrer, pour le lecteur intéressé, les processus de création mathématique. Pólya met souvent l'accent sur les « questions de recherche » pour aider les apprentimathématiciens souhaitant résoudre des problèmes. L'équipe de Varga, s'adressant aux enseignants, expérimente plusieurs formes d'écriture qui cherchent à offrir un aide pour le choix et la mise en ordre des problèmes, et conseille des questions à poser en classe pour guider le processus de découverte des élèves. Mais on peut chaque fois reconnaitre une attention particulière accordée à la mise en ordre des problèmes.

On peut donc conclure que dans la tradition hongroise, il existe une réflexion approfondie pour développer un genre d'écriture qui permet de rendre compte d'une pratique pédagogique basée sur un enchaînement complexe et sophistiqué de problèmes. 
Une analyse plus approfondie de cette pratique nécessitera avant tout une étude plus détaillée des textes présentés, et plus particulièrement la caractérisation de la structure des séries de problèmes. En outre, nous pourrons prendre en considération un ensemble élargi des sources. D'une part, vu qu'il s'agit d'une période quasi-contemporaine, des sources orales sont aussi disponibles : entretiens avec des enseignants par exemple, ou observations des classes d'aujourd'hui des disciples de Varga (dont la pratique n'est sans doute pas identique avec celle des années 1970, mais on peut supposer une certaine continuité). D'autre part, on pourra considérer des notes des enseignants préparant leurs cours (qui comprennent, dans de nombreux cas d'enseignants hongrois, des séries de problèmes). En effet, ces sources du type intermédiaire, à la fois écrits mais servant de supports pour une pratique orale, pourraient aider à mieux comprendre comment la pratique d'enseignement de la tradition hongroise est composée : d'un travail de rédaction préalable, la construction des séries de problèmes; et d'une pratique orale improvisée, une adaptation des séries de problèmes aux réactions des élèves, et un discours dialogique entre la classe et l'enseignant.

\section{Références}

Andrews, P. (2003). Opportunities to learn in the Budapest Mathematics Classroom. International Journal of Science and Mathematics Education 1, 201-225.

Andrews, P. \& Hatch, G. (2001). Hungary and its caracteristic pedagogical flow. In J. Winter (Ed.), Proceedings of the British Society for Research into Learning Mathematics, 21(2), 26-40. Consulté sur http://www.bsrlm.org.uk/IPs/ip21-2/BSRLM-IP-21-2-Full.pdf (29.10.15).

C. Neményi, E., Göndöcs, L., Merő, L., Merő, L., \& Varga, T. (1978), Kézikönyv a matematika 1. osztályos anyagának tanításához. Budapest : Tankönyvkiadó.

C. Neményi, E., \& Varga, T. (1978). Matematika munkalapok. Budapest : Tankönyvkiadó.

Császár, Á. (2005). Education and Research in Mathematics. In Horváth, János (Ed.), A Panorama of Hungarian Mathematics in the Twentieth Century (pp. 555-562). Bolyai Society Mathematical Studies 14. Budapest Berlin: János Bolyai Mathematical Society Springer-Verlag.

d'Enfert, R., \& Kahn, P. (Eds.) (2011). Le temps des réformes. Disciplines scolaires et politiques éducatives sous la Cinquième République (années 1960). Grenoble : Presses Universitaires de Grenoble.

Eglesz, I., Kovács, Cs., \& Sztrókayné Földvári V. (1979). Matematika általános iskola 5. Budapest : Tankönyvkiadó.

Eglesz, I., Kovács, Cs., Radnainé Szendrei, J., \& Sztrókayné Földvári, V. (1981). Kézikönyv a matematika 5. osztályos anyagának tanításához. Budapest : Tankönyvkiadó.

Frank, T. (2012). Teaching and Learning Science in Hungary, 1867-1945: Schools, Personalities, Influences. Science \& Education, 21(3), 355-380.

Gallai, T., \& Péter, R. (1949). Matematika a középiskolák 1. osztálya számára. Budapest : Tankönyvkiadó Nemzeti Vállalat.

Gimes, Gy. (Ed.) (1980). Összefoglaló feladatgyüjtemény matematikából. Budapest : Tankönyvkiadó.

Gispert, H. (2008). L'enseignement des mathématiques au XXe siècle dans le contexte français. Site "CultureMaths", article publié le 23 mars 2008. Paris : ENS Degesco. http://www.math.ens. fr/culturemath/histoire $\% 20$ des $\% 20$ maths/htm/Gispert08-reformes/Gispert08.htm (consulté 19.10.2015).

Gispert, H. (2010). Rénover l'enseignement des mathématiques, la dynamique internationale des années 1950. In. R. d'Enfert \& P. Kahn (Eds.) (2010), En attendant la réforme. Disciplines scolaires et politiques éducatives sous la Quatrième République (pp. 135-147), Grenoble : Presses universitaires de Grenoble.

Glaymann, M., \& Varga, T. (1973). Les Probabilitiés á L'école. Paris : CEDIC.

Gosztonyi, K. (2014). Séries de problèmes. L'exemple des Jeux avec l'infini de Rózsa Péter. Bulletin APMEP, 507, 25-31. 
Gosztonyi, K. (à paraître). Mathematical culture and mathematics education in Hungary in the XXth century. In B. Larvor, (Ed.), Mathematical Cultures. Springer.

Gurka, D. (2001). Kalmár László szerepe Lakatos Imre matematikafilozófiájának alakulásában. In G. Palló (Ed.), Recepció és kreativitás. Consulté sur http://www . phil-inst . hu/recepcio/htm/ 3/310_belso.htm

Hersch, R., \& John-Steiner, V. (1993). A Visit to Hungarian Mathematics, The Mathematical Intelligencer, 15(2), 13-26.

Kalmár, L. (1942). A matematikai egzaktság fejlődése a szemlélettôl az axiomatikus módszerig. In S. Karácsony (Ed.), A másik ember felé. Debrecen: Exodus.

Kalmár, L. (1967). Foundations of Mathematics: Whither Now? In I. Lakatos (Ed.), Problems in the Philosophy of Mathematics (pp. 186-194). Amsterdam: North-Holland Publishing Co.

Kalmár, L. (1986). Integrállevél. (A. Varga, Ed.), Budapest: Gondolat.

Kilpatrick, J. (2012). The new math as an international phenomenon. ZDM, 44(4), 563-571.

Kontra, Gy. (1992). Karácsony Sándor. Budapest: Országos Pedagógiai Könyvtár és Múzeum.

Kovács, Cs., Sz. Földvári V., \& Szeredi É. (1980). Matematika. Általános iskola 7. osztály. Budapest: Tankönyvkiadó.

Lakatos, I. (1976). Proofs and Refutations. Cambridge : Cambridge University Press.

Máté, A. (2006). Árpád Szabó and Imre Lakatos, Or the relation between history and philosophy of mathematics. Perspectives on Science, 14(3), 282-301.

Máté, A. (2008). Kalmár László és Péter Rózsa - matematikusok a filozófiáról. In P. G. Szabó (Ed.), Kalmárium II (pp. 56-71). Szeged: Polygon.

Pálfalvi, J. (2007). Egy szép példa Varga Tamástól. In M. Halmos, \& J. Pálfalvi (Eds.), Matematikatanár-képzés - matematikatanár-továbbképzés (pp. 3-4). Budapest: Nyitott Könyvmúhely.

Péter, R. (1977). Jeux avec l'infini (G. Kassai trad.). Paris : Éditions du Seuil. (Ouvrage original publié en 1944 sous le titre Játék a végtelennel. Budapest, Dante Könyvkiadó).

Péter, R. (2004). Matematika és múvészet - nem két ellentétes pólus. In S. Róka \& D. Valcsicsák (Eds.), A jövó a számtantudósoké. Magyar szerzók írásai a matematikáról (pp. 195-213). Budapest: Noran.

Pólya, G. (1965). Comment poser et résoudre un problème (C. Mesnage trad.). Paris : Dunod. (Ouvrage original publié en 1945 sous le titre How to solve it. Princeton : Princeton University Press).

Prékopa, A., Kiss, E., ,Staar, Gy., \& Szenthe, J. (2004). Bolyai-emlékönyv. Budapest : Vincze Kiadó.

Programme et enseignement des mathématiques à l'école élémentaire (1970). Consulté sur http://www. formapex.com/repertoires/550-programmes-textes-officiels (29.10.15).

Rényi, A. (2005). Ars Mathematica. Rényi Alfréd összegyújtött írásai. Budapest: Typotex.

Scharnitzky, T., \& Török, T. (2002). Emlékek Varga Tamásról. In M. Halmos \& J. Pálfalvi (Eds.), Matematikatanár-képzés - matematikatanár-továbbképzés 6 (pp. 3-8). Budapest: Múszaki Könyvkiadó.

Servais, W., \& Varga, T. (Eds.) (1971). Teaching school mathematics. A Unesco source book. Middelsex: Penguin Books.

Szabó, M. (2013). Karácsony Sándor nyelvfelfogásának hatása Kalmár László korai matematikafilozófiájára. In Zvolenszky et al. (Eds.), Nehogy érvgyúlölók legyünk. Tanulmánykötet Máté András 60. születésnapjára (pp. 164-173). Budapest: L'Harmattan.

Szabó, P. G. (Ed.) (2005). Kalmárium. Szeged: Polygon.

Szebenyi, P. (Ed.) (1978). Az általános iskolai nevelés és oktatás terve. Budapest: OPI.

Varga, T. (1975). Kandidátusi értekezés. (Thèse inédit). Académie des Sciences de la Hongrie, Budapest.

Varga, T. (1982). New topics for the elementary school math curriculum. In. Th. C. O'Brien (Ed.), Toward the 21st Century in Mathematics Education (pp. 12-34). Teachers' Center Project, Southern Illionis University at Edvardsville.

Varga, T. (1987). Az egyszeregy körül. Kritika, 25(12), 28-31. 


\section{SHS Web of Conferences}

\section{Annexe I. (C. Neményi - Göndöcs et al. 1978, p.98-99 et annexes.)}
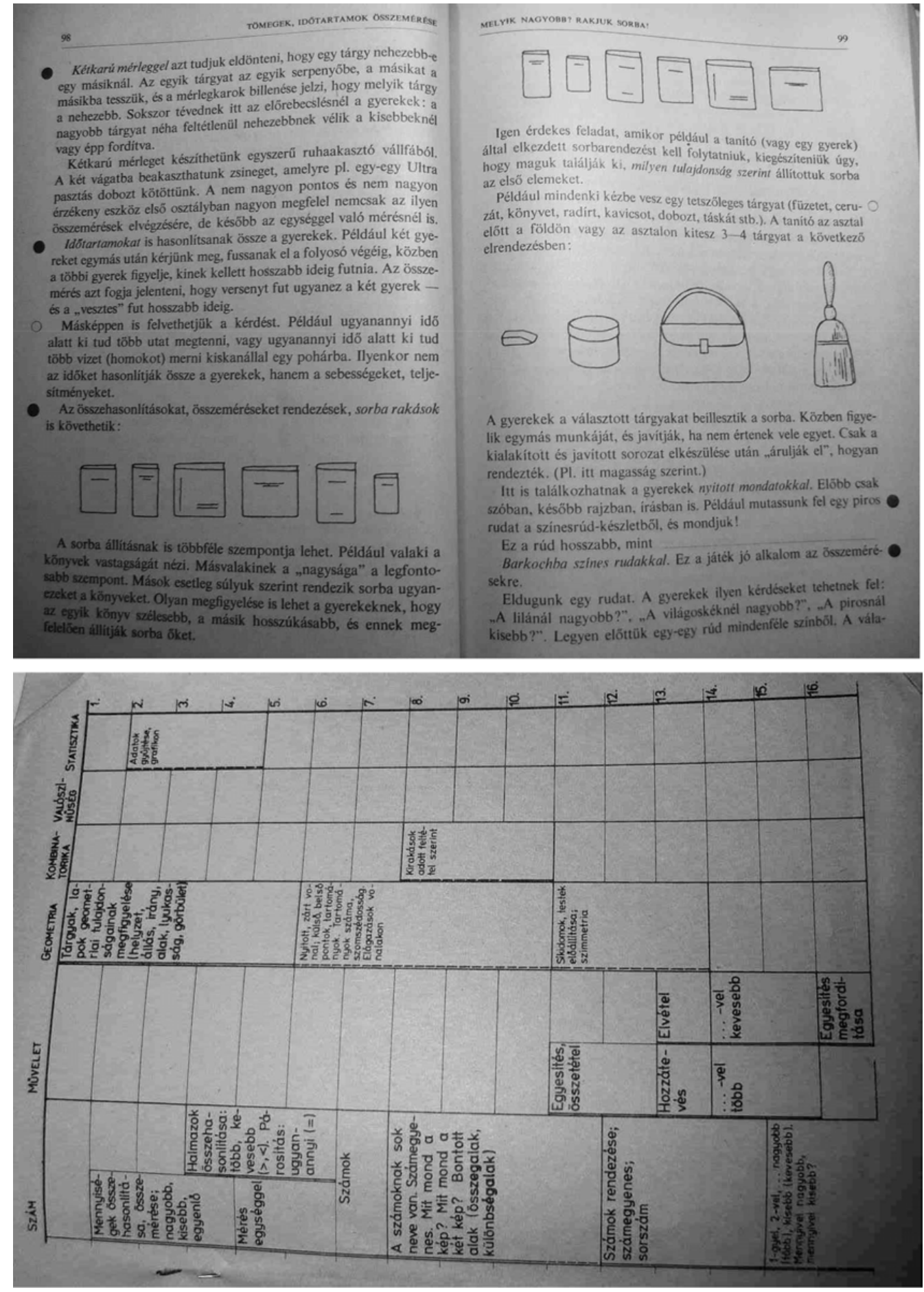
Les séries de problèmes, un genre au carrefour des cultures

Annexe II. (Eglesz-Kovács-Sztrókayné 1979, p. 215-217.)

Milyen messre van a tollhegy a tintapacnitol?

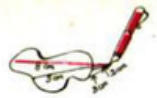

Zungi: Megint napon sokféteképpen mérhetnénk, de az elôbb abban a pires vonat menth kell metrni. A tollthegy ts a pacni tivolsiga $8 \mathrm{~cm}$.

$\pi$

Aztrt ez meg sines egtszen rendben, mondta Gergó. mert ha a tollhegy rajta van a pacin, akkor is azt modod, hogy a tollhegy es a paca tívolsaga $2 \mathrm{~cm}$ ?

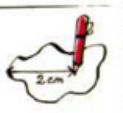

valóban $2 \mathrm{~cm}-$ nek merem, de ha rajta van a pací. akkor nyilvin sokkal kơzelebb van hozza

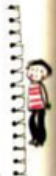

Milyen messze van a $P$ pont az e egyenestôn?

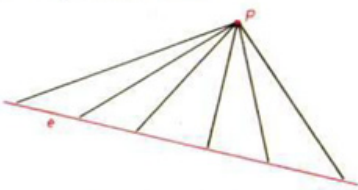

Most mír en is bajban vagyok, mondta Zsuzsi. Nem todom megtalate bbi pontjitt, hiven akirmeddis is rajzolhatnink Af. eqgenest, sohase lenne vege. Akirmelyik szakasz menten mérnenk 3. mindig lehetne egy móg hosszabb szakasst találni. Azt méges mondhatjuit IIf hogy a pont az egyenestof akär $100 \mathrm{~m}$-net is messrebb van, hiszen litur hogy elég kơzel van horzi.

Robi: Mirpedig ha rapaskodsz az elóbbi megallapodishoz, ant kell mondanod, hogy a pontnak is az ezyenesnek akírmilyen nagy is lehet a tavolatei





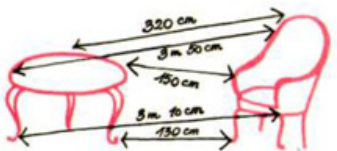

Milyen messze van a fotel az asztaltón?

A gyerekek mérószalaggal mértek

Q Zsursi: Most kinek van igaza? Ahányan mérünk, annyi eredmény kapunk. Honnan tudjam, hogy az asstal, illetve a stek melyik pontjiłho tegyem a mérószalagot?

Robi: Végezzūnk egy csomó mérést, ts ezek átlagát nevezzūk az aștal a wék távolsaigának! a legnagyobb mogy a legkisebb mertsi eredmenyt fogadjuk el az astal ts a srék távolságinak.

Lussi: Szerintem mindegy, melyik javaslatot fogadjuk el, az a lényeg. Thy hat to ax antal theole dolunk, vagyis megällapodidsumk egyértelmä lezjen. A gyerekek megalllapodtak abban, hogy a legnagyobb szakasz hosszat neverik rolsignak! Bár minden lehetstges szakaszt megmerni nem lehet, de biztosan van tottak leghosszabb (esetleg tôbb is).

Az asztal ts a szetk tívolsagit ennek alapjän $3 \mathrm{~m} 50 \mathrm{~cm}$-nek mérték.

\section{$\pi$ in}

itt is:

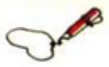

witi is:

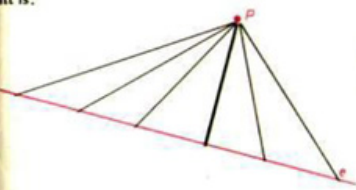

It eppen a meróeges szakasz legrôvidebb.

Sót még Gergónek is igaza van, a tollhegy es a paca távolsaga most $0 \mathrm{~cm}$.

Ex persze nem azt jelenti, hogy nincs távolsága, hiszen a 0 is a számok egyike.

Illapitsuk meg a ket ponthalmaz tívolsight!

- $\quad$ A piros pontokat kösak óssze a lekete pontokkal minden lehetskges módon.

Hány szakaszt kapunk?

ndegyiket megmerjak.

A kèt ponthalmaz pontjait czymással ôssrekötố szakuszok kôzôl a legroóvidebt tasz hosszit nevezzalk a két ponthalmax tívolsiganaak 


\section{Annexe III (Kovács, Sz. Földvári \& Szeredi 1980 p. 256-257.)}



123. Trouve parmi les triples de nombres ceux qui déterminent un triangle rectangle, acutangle et obtusangle.

124. Quelle est la distance du point $\mathrm{P}(3 ; 4)$ de l'origine du repère orthogonal?

125. Quelle est la distance du point $\mathrm{P}(5 ;-12)$ de l'origine du repère orthogonal ?

126. Quelle est la distance du point $\mathrm{P}(-6 ;-8)$ de l'origine du repère orthogonal ?

127. Quelle est la distance de l'origine du repère orthogonal du point dont la distance d'un axe est 3 et de l'autre axe est 4 unités ? Combien existe-t-il de tels points ?

128. Quelle est la distance entre les points A $(3 ; 6)$ et B $(3 ; 11)$ ?

129. Quelle est la distance entre les points A $(4 ; 9)$ et B $(11 ; 9)$ ?

130. Quelle est la distance entre les points A $(2 ; 1)$ et B $(6 ; 4)$ ?

131. Quelle est la distance entre les points A $(-1 ;-1)$ et B $(3 ; 2)$ ?

132. Quelle est la distance entre les points A $(-3 ;-2)$ et B $(9 ; 3)$ ?

133. Les coordonnées du point A sont $\left(x_{1} ; y_{1}\right)$, du B sont $\left(x_{2}\right.$; $y_{2}$ ). Cherche une formule qui permet de calculer la distance entre les deux points.

134. Dessine un triangle, et divise chaque côté en a) 3 parties égales; b) 5 parties égales; c) des parties dont la proportion est de 2 à 3.

135. Construis un triangle dont le périmètre est donné, et dont on sait que la proportion de ces côtés est $3: 4: 5$.

136. Il est donné un triangle et un segment. Construis un triangle semblable à celui qui est donné, et dont l'un des côtés soit égal au segment donné.
114. Dans un triangle rectangle, les deux côtés de l'angle droit mesurent $4 \mathrm{~cm}$ et $7 \mathrm{~cm}$. Quelle est la longueur de l'hypoténuse ?

115. Dans un triangle rectangle, l'hypoténuse mesure 11 $\mathrm{cm}$, un des côtés de l'angle droit mesure $4 \mathrm{~cm}$. Quelle est la longueur de l'autre côté ?

116. Dans un triangle rectangle, deux côtés mesurent 2 $\mathrm{cm}$ et $3 \mathrm{~cm}$. Combien peut mesurer le troisième côté ?

117. Détermine le côté du losange. a) e $=8 \mathrm{~cm}, \mathrm{f}=6 \mathrm{~cm}$ ; b) $\mathrm{e}=10 \mathrm{~cm}, \mathrm{f}=24 \mathrm{~cm}$; c) $\mathrm{e}=16 \mathrm{~cm}, \mathrm{f}=30 \mathrm{~cm}$.

118. Détermine la diagonale manquante du losange. a) $\mathrm{a}=17 \mathrm{~cm}, \mathrm{f}=16 \mathrm{~cm} ; \mathrm{b}) \mathrm{a}=25 \mathrm{~cm}, \mathrm{f}=14 \mathrm{~cm}$.

119. Détermine la hauteur du triangle isocèle si $a) a=6$ $\mathrm{cm}, \mathrm{b}=8 \mathrm{~cm} ; \mathrm{b}) \mathrm{a}=16 \mathrm{~cm}, \mathrm{~b}=17 \mathrm{~cm}$.

120. Kati a dessiné deux triangles rectangles non isométriques. Dans chacun des deux, il y a un côté mesurant $3 \mathrm{~cm}$ et un autre mesurant $4 \mathrm{~cm}$. Combien sont les périmètres et les aires de ces deux triangles ?

121. On a désigné les côtés de l'angle droit des triangles rectangles avec $a$ et $b$, et leur hypoténuse avec $c$. Remplis le tableau.

122. Les triples de nombres ci-dessous sont chacun les mesures des côtés d'un triangle. Quels sont parmi eux les mesures des côtés d'un triangle rectangle ?

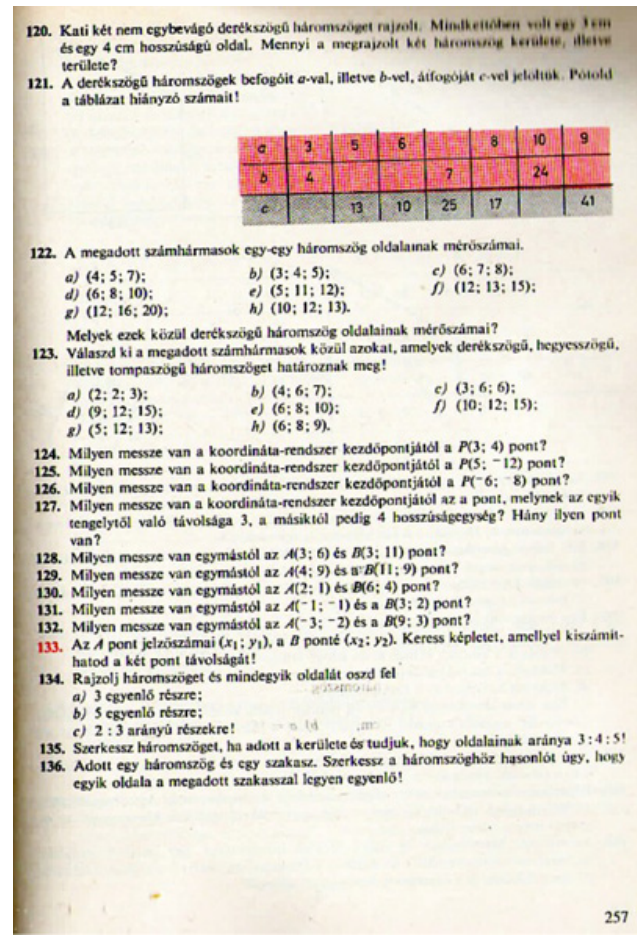

